\title{
Polynomial Induction and Length Minimization in Intuitionistic Bounded Arithmetic
}

\author{
Morteza Moniri \\ Department of Mathematics, Shahid Beheshti University, Evin, Tehran, Iran. \\ AND: Institute for Studies in Theoretical Physics and Mathematics (IPM), \\ P.O. Box 19395-5746, Tehran, Iran. \\ email: ezmoniri@ipm.ir
}

\begin{abstract}
It is shown that the feasibly constructive arithmetic theory IPV does not prove (double negation of) LMIN(NP), unless the polynomial hierarchy CPV-provably collapses. It is proved that PV plus (double negation of) LMIN(NP) intuitionistically proves PIND $($ coNP). It is observed that $\mathrm{PV}+\mathrm{PIND}(\mathrm{NP} \cup \mathrm{coNP})$ does not intuitionistically prove NPB, a scheme which states that the extended Frege systems are not polynomially bounded.
\end{abstract}

2000 Mathematics Subject Classification: 03F30, 03F55, 03F50, 68Q15.

Key words: Kripke model; Polynomial Induction; Length Minimization; IPV; Extended Frege System.

\section{Introducing Classical and Intuitionistic Bounded Arithmetic}

The theory $P V$ is an equational theory of polynomial time functions introduced by Stephen Cook, $(P V)^{i}$ is its extension to intuitionistic first-order logic and $I P V$ is the intuitionistic theory of $P V$ plus polynomial induction on NP formulas. Here an NP formula is a formula equivalent to an atomic formula (in the language of $P V$ ) prefixed by a bounded existential quantifier (see $[\mathrm{CU}]$ ). Also, the instance of the Polynomial Induction $P I N D$ with respect to a distinguished free variable $x$ on a formula $\varphi(x)$ is the sentence

$$
\left[A(0) \wedge \forall x\left(A\left(\left\llcorner\frac{x}{2}\right\lrcorner\right) \rightarrow A(x)\right)\right] \rightarrow \forall x A(x)
$$


The NP formulas represent precisely the NP relations in the standard model. coNP formulas are defined dually. The theory $(P V)^{i}$ proves the Principle of Excluded Middle for atomic formulas (of $P V$ ).

The classical deductive closure of $P V$ is usually denoted $P V_{1} . C P V$ is the classical version of $I P V$.

In the following, the notation $\equiv_{i}$ between two sets of formulas is used to show that they have the same intuitionistic consequences. Also, $\vdash_{i}$ denotes provability in intuitionistic (first-order) logic.

If $\Gamma$ is a set (collection) of formulas, $\neg \Gamma$ denotes the set of formulas of the form $\neg \varphi$ with $\varphi \in \Gamma$.

For the definition of Kripke models of intuitionistic bounded arithmetic and basic results about them, see [M2] and [B2]. The general results on intuitionistic logic and arithmetic, and also Kripke models, can be found in [TD].

For a set $T$ of sentences, a $T$-normal Kripke model is a Kripke model in which all the worlds (classically) satisfy $T$.

\section{Polynomial induction versus length minimization}

In this section we work in the language of $P V$. Also, $(P V)^{i}$ is the underlying theory for all intuitionistic theories we will mention.

The instance of the length minimization $L M I N$ with respect to a distinguished free variable $x$ on a formula $\varphi(x)$ is the sentence

$$
\exists x \varphi(x) \rightarrow\left[\varphi(0) \vee \exists x\left(\varphi(x) \wedge\left(\forall z \leqslant\left\llcorner\frac{x}{2}\right\lrcorner\right) \neg \varphi(z)\right)\right] .
$$

We will compare intuitionistic schemes of polynomial induction and length minimization on NP formulas. By $\neg \neg L M I N(\mathrm{NP})$, we denote the intuitionistic theory axiomatized by $P V$ plus the set of all doubly negated instances of $L M I N$ on NP formulas.

Proposition 2.1 If $\mathcal{K} \Vdash \neg \neg L M I N(\mathrm{NP})$ is linear, then the union of the worlds in $\mathcal{K}$ satisfies $C P V$.

Proof First note that $(P V)^{i}$ is contained in the theory $\neg \neg L M I N(\mathrm{NP})$ by our assumption, so each of the nodes in $\mathcal{K}$ forces $(P V)^{i}$. But $(P V)^{i}$ is a universal theory, so each node satisfies the classical deductive closure of $(P V)^{i}$, i.e. $P V_{1}$. Therefore, the union of the worlds in $\mathcal{K}$ satisfies $P V_{1}$. Recall that $C P V \equiv_{c} P V+P I N D(\mathrm{coNP})$. So, it is enough to show that PIND(coNP) holds in the union. Assume that the union does not satisfy $P I N D(A(x))$, for some coNP formula $A$. Here, it is possible that $A$ has other free variables, besides the one explicitly shown. Let $A$ be of the form $\forall y B(y, x)$, where $B$ is a quantifier-free formula. Assume $C$ to be the formula $\exists y \neg B(y, x)$, an NP formula. There would exist a node $M_{\gamma}$ present in $\mathcal{K}$ and some $a \in M_{\gamma}$, such that (a) $M_{\gamma} \vDash \neg C(0) \wedge C(a)$ and (b) the union satisfies $\forall x\left(\neg C\left(\left\llcorner\frac{x}{2}\right\lrcorner\right) \rightarrow \neg C(x)\right)$ (here we have replaced all other free 
variables of $C$ with parameters from $M_{\gamma}$ ). We have $\gamma \Vdash C(a)$ (because forcing and truth of $C(a)$ are equivalent) and $\gamma \Vdash \neg C(0)$ (since the union satisfies $\forall y B(y, 0)$ ). Therefore, by $\mathcal{K} \Vdash \neg \neg L M I N(\mathrm{NP})$, we get

$$
\gamma \Vdash \neg \neg \exists x\left(C(x) \wedge \forall z \leq\left\llcorner\frac{x}{2}\right\lrcorner \neg C(z)\right) .
$$

In particular, for some $\delta \geq \gamma$ and some (necessarily nonzero) $d \in M_{\delta}, \delta \Vdash C(d) \wedge \forall z \leq$ $\left\llcorner\frac{d}{2}\right\lrcorner \neg C(z)$.

Therefore, the union satisfies $\neg C\left(\left\llcorner\frac{d}{2}\right\lrcorner\right)$. On the other hand, by $\delta \Vdash C(d), M_{\delta} \vDash C(d)$. Hence, the union satisfies $C(d)$. The combination of these two leads to a contradiction to (b).

It is known that $C P V$ proves $L M I N(\mathrm{NP})$. Here, we show that even $\neg \neg L M I N(\mathrm{NP})$ is not provable in $I P V$ under some plausible complexity-theoretic assumption.

Theorem 2.2 IPV $\nvdash \neg \neg L I N(\mathrm{NP})$, unless $C P V=P V_{1}$.

Proof Assume $I P V \vdash \neg \neg L M I N(\mathrm{NP})$. Any $\omega$-chain of (classical) models of $C P V$ can be considered as a Kripke model of $I P V$ whose underlying accessibility relation has order type $\omega$ (the proof is straightforward, see [M2]). Now, by the assumption, this model forces $\neg \neg L M I N(\mathrm{NP})$ as well, hence by 2.1 , the union of its worlds should satisfy $C P V$. This shows that $C P V$ is an inductive theory. Hence, using the well-known characterization of the inductive theories (see e.g. [CK, Th. 3.2.3]), $C P V$ should be $\forall_{2}$. Now, using $\forall_{2^{-}}$ conservativity of $C P V$ over $P V_{1}$ (see [B1, Th. 5.3.6 and Coro. 6.4.8]), we get $C P V \equiv P V_{1}$ which is what we wanted.

It is known that, under the assumption $C P V=P V_{1}$, the polynomial hierarchy $C P V$ provably collapses, see [K, Theorem 10.2.4].

Here we state a small result which is a converse to Proposition 2.1.

Proposition 2.3 If $\mathcal{K} \Vdash(P V)^{i}$ and the union of the worlds in any path of $\mathcal{K}$ satisfies $C P V$, then $\mathcal{K} \Vdash P I N D(\operatorname{coNP})$.

Proof Note that a coNP formula is forced at a node $\alpha$ of a Kripke model of $P V$ if and only if it is satisfied in the union of the worlds in any path above $\alpha$.

Theorem 2.4PV+LMIN(NP) $\vdash_{i} P I N D(\operatorname{coNP})$.

Proof The proof is similar to the one for Proposition 2.1. Let $\mathcal{K} \Vdash P V+L M I N(\mathrm{NP})$. Consider an arbitrary coNP formula $A(x, \bar{y})$. Assume $\alpha$ is an arbitrary node in $\mathcal{K}$ and $\bar{b} \in M_{\alpha}$. Suppose also that $\alpha \Vdash A(0, \bar{b}) \wedge \forall x\left(A\left(\left\llcorner\frac{x}{2}\right\lrcorner, \bar{b}\right) \rightarrow A(x, \bar{b})\right)$. We shall show that $\alpha \Vdash \forall x A(x, \bar{b})$. If for every $\beta \geqslant \alpha, M_{\beta} \vDash A(x, \bar{b})$, then we have $\alpha \Vdash \forall x A(x, \bar{b})$. Suppose not. Assume $\eta \geqslant \alpha$ does not have the mentioned property. Let $A(x, \bar{b})$ be of the form $\forall z B(x, z)$, where $B$ is a quantifier-free formula. Assume $C(x)$ to be the formula $\exists z \neg B(x, z)$, an NP formula.

We have $M_{\eta} \nVdash C(0)$ and $M_{\eta} \Vdash C(a)$ for some $a \in M_{\eta}$. Hence, by $\mathcal{K} \Vdash L M I N(\mathrm{NP})$, we get $\eta \Vdash \exists x\left(C(x) \wedge \forall z \leq\left\llcorner\frac{x}{2}\right\lrcorner \neg C(z)\right)$. Clearly, such a node $\eta$ forces $\operatorname{PIND}(A(x, \bar{b}))$. 
Corollary 2.5 $\neg \neg L M I N(\mathrm{NP}) \vdash_{i} P I N D(\mathrm{coNP})$.

Proof Using the general equivalence $\neg \neg(A \rightarrow B) \equiv_{i}(A \rightarrow \neg \neg B)$, it is easy to see that in $(P V)^{i}, \neg \neg P I N D(A(x)) \equiv_{i} P I N D(A(x))$ for any coNP formula $A$. Now, Theorem 2.4 immediately implies what we want.

\section{Unprovability of $N P B$ in $P V+P I N D(N P \cup$ coNP $)$}

Let $f$ be a one-place function symbol of $I P V$. Suppose $f$ is provably an increasing function and provably dominates any polynomial growth rate function. Let $N P B(f)$ be the formula

$$
\forall x \exists y\left(x \leqslant y \wedge T A U T(y) \wedge \forall z\left(z \leqslant f(y) \rightarrow \neg z \vdash_{e \mathcal{F}} y\right)\right) .
$$

Here TAUT $(y)$ states that $y$ is the Godel number of a propositional tautology and $z \vdash_{e \mathcal{F}} y$ states that $z$ is the Godel number of an extended Frege proof of the formula coded by $y$, see $[\mathrm{K}]$ for the definitions. In the sequel, we fix $f$ and write $N P B$ instead of $N P B_{f}$.

Cook and Urquhart [CU, Th. 10.16] proved that, IPV $\nvdash N P B$ using their characterization of provably total functions of $I P V$. Krajicek and Pudlak proved that $P V_{1} \nvdash N P B$ by constructing a chain of models of $P V_{1}$ such that the union of its worlds does not satisfy $N P B$, see [K]. Buss [B2] used the model theoretic method of Krajicek and Pudlak and also used Kripke models to show that $I P V^{+} \nvdash \neg \neg N P B$. The theory $I P V^{+}$ which was introduced by Buss [B2] apparently is stronger than $I P V$ and is sound and complete with respect to $C P V$-normal Kripke structures. Here, we use a simple model theoretic proof to show $P V+P I N D(N P \cup \operatorname{coNP}) \nvdash_{i} N P B$. This theory is actually equivalent to the theory $I P V^{*}$, which is by definition the intuitionistic theory axiomatized by $P V+P I N D(N P \cup \neg \neg N P)$, originally mentioned in [CU] and studied in [M1]. The reason is that, by [M2, Theorem 2.3], $P V+P I N D(\operatorname{coNP}) \equiv_{i} P V+P I N D(\neg \neg \mathrm{NP})$. The proof of [M1, Theorem 2.5] actually shows that $I P V^{+} \nvdash I P V^{*}$ unless $C P V=P V_{1}$.

$N P B$ is intuitionistically equivalent to $\forall x \exists y \forall z \mathrm{NPB}_{\mathrm{M}}$. Here $N P B_{\mathrm{M}}$ is an atomic formula formalizing " $x \leqslant y$, and $z$ is a satisfying assignment of $\mathrm{y}$, and if $z \leqslant f(y)$ then $z$ is not an extended Frege proof of $y$ ". Below, we work with this form of NPB.

\section{Theorem 3.1 $P V+P I N D(N P \cup \operatorname{coNP}) \nvdash_{\mathrm{i}} \mathrm{NPB}$}

Proof Let $M \vDash P V_{1}+\neg N P B$ be countable. Such a model exists by the above mentioned result of Krajicek and Pudlak. Extend $M \Sigma_{1}^{b}$-elementarily to a model of $C P V$, for existence of such a model see [K, Theorem 7.6.3]. Now, consider the obvious two-node Kripke model. It is easy to see that this Kripke model forces $P V+P I N D(\mathrm{NPUcoNP})$. On the other hand this model does not force the prenex sentence $N P B$ since otherwise its root-model would satisfy this sentence, which is a contradiction.

Note that the Kripke model constructed in the above Theorem forces $I P V^{+}$if and 
only if $M \vDash C P V$, see [M1, Theorem 2.2].

Here we just mention that, by the following theorem, which is the main result of [CU], all prenex consequences of $I P V$ are already provable in $(P V)^{i}$ :

Theorem 3.2 (Cook and Urquhart, [CU])

(i) If $f$ is a polynomial time computable function then $f$ is $\Sigma_{1}^{b+}$-definable in $I S_{2}^{1}$.

(ii) If $I S_{2}^{1} \vdash \forall \bar{x} \exists y \phi(\bar{x}, y)$ then there is a polynomial time computable function $f$ such that $I S_{2}^{1} \vdash \forall \bar{x} \phi(\bar{x}, f(\bar{x}))$.

Note that, in part (ii) above, the function symbol $f$ does not belong to the language of $I S_{2}^{1}$; however by part (i), it can be expressed in the language.

\section{Acknowledgements}

My thanks to Shahid Beheshti university, Tehran, Iran, for financial support.

\section{References}

[A] J. Avigad, Interpreting Classical Theories in Constructive Ones, Journal of Symbolic Logic, 65 (2000) 1785-1812.

[B1] S. R. Buss, Bounded Arithmetic, Bibliopolis, 1986.

[B2] S. R. Buss, On Model Theory for Intuitionistic Bounded Arithmetic with Applications to Independence Results, in: Feasible mathematics, eds S. R. Buss and P. J. Scott, 1990, 27-47, Birkhauser.

[CK] C.C. Chang and J. Keisler, Model theory, North-Holland, 1990.

[CU] S. A. Cook and A. Urquhart, Functional Interpretations of Feasibly Constructive Arithmetic, Annals of Pure and Applied Logic, 63 (1993), 103-200.

[K] J. Krajicek, Bounded Arithmetic, Propositional Logic, and Complexity Theory, Cambridge University Press, 1995.

[M1] Morteza Moniri, On Two Questions About Feasibly Constructive Arithmetic, Mathematical Logic Quarterly, 49 (2003) 425-427.

[M2] Morteza Moniri, Comparing Constructive Arithmetical Theories Based on NP-PIND and coNP-PIND, Journal of Logic and Computation, 13 (2003) 881-888.

[TD] A. S. Troelstra and D. van Dalen, Constructivism in Mathematics, v.I, NorthHolland, 1988. 